\title{
Le siècle du vieillissement
}

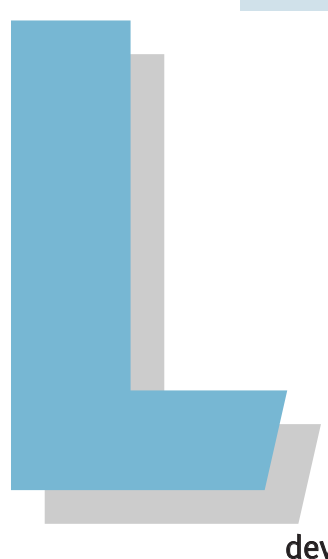
qu'il recouvre réellement, en trois idées simples.

Rappelons d'abord que le vieillissement, c'est-à-dire l'augmentation de la proportion des personnes âgées dans une population, est inéluctable en France et en Europe, au XXIe siècle, pour deux raisons ${ }^{1}$.

D’une part, les territoires cités connaissent un vieillissement «par le bas » en raison d'une fécondité et d'une natalité très abaissées. Phénomène toutefois moins intense en $\mathrm{F}$ rance par $\mathrm{r}$ apport à la mo yenne européenne.

D’autre part, ces territoir es vivent un autre vieillissement, quant à lui heur eux, le vieillissement « par le haut ", résultat de l'augmentation de l'espér ance de vie des personnes âgées. $L$ e caractère heureux de ce vieillissement est confirmé par la situation inv erse de pays d'Europe orientale dont l'espérance de vie a diminué ces dernières décennies.

Si l'on peut considérer, sauf catastrophe, que le vieillissement « par le haut » est inéluctable, il n'en est pas moins vrai que le vieillissement « par le bas » peut connaître des intensités for $t$ variables et des conséquences diverses, sachant par ex emple qu'un printemps démographique modifierait considérablement la donne. Il est ainsi clair que la situation des Etats-

Unis, avec une fécondité au seuil de remplacement des générations, supérieure de $40 \%$ à celle de l'E urope, n'est en rien comparable.

Le vieillissement conduit à des effets de structur e. Mais l'analyse des évolutions de sociétés doit aussi considérer les flux de personnes âgées, ce que j’appelle la gér ontocroissance ${ }^{2}$, soit l'augmentation du nombre des personnes âgées.

Deuxièmement, contrairement aux discours dominants et répétitifs sur la croissance exponentielle de la population mondiale, cette dernièr e, comme nous l'avons annoncé depuis longtemps, ne se produira pas. Le XXIe siècle sera, pour l'ensemble du monde, le siècle du vieillissement sous l'effet d'une fécondité déjà en dessous du seuil de remplacement pour plus de la moitié des populations et d'une décélér ation démographique souvent rapide.

Troisièmement, les tr ois phénomènes, vieillissement "par le bas ", vieillissement « par le haut " et gérontocroissance évoluent de façon extrêmement div ersifiée selon les territoires, d'autant que s'ajoutent aux effets du mouvement naturel ceux du mouv ement migratoire. Par exemple en France, lors de la dernièr e période intercensitaire, le département du Var a cumulé gérontocroissance et vieillissement, autrement dit hausse du nombre de personnes âgées et de leur proportion. À l'opposé, P aris a connu deux évolutions inverses sous l'effet de sa structure démographique atypique et de son système migratoire. En Creuse, les données montrent d'une part une proportion croissante des personnes âgées et, en même temps, une baisse du nombr e de personnes âgées en $r$ aison d'un contexte de dépopulation.

Ainsi, derrière le mot dev enu couramment usité de vieillissement, se cachent des phénomènes diversifiés, portés par des combinaisons de facteurs div ers, des perspectives différentes en fonction de la fécondité à venir, et des réalités territoriales forts disparates selon l'intensité des phénomènes et des facteurs les expliquant.

C'est pourquoi Population \& Av enir a décidé d'approfondir davantage cette question en organisant le colloque scientifique européen qui fait l'objet de l'annonce ci-dessous.
1. Sans oublier deux autres facteurs les effets de l'histoire démographique et des systèmes migratoires.

2. Cf. notamment Gérard-François Dumont, " Vieillissement et gérontocroissance », Population \& Avenir, $n^{\circ} 663$, mai-juin 2003.

\section{Sous l'égide de la \\ DATAR En partenariat avec

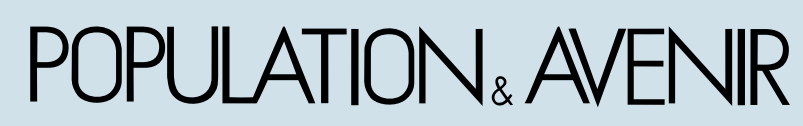

